\title{
Functional role of the nicotinic arm of the acetylcholine regulatory axis in human B-cell lines
}

This article was published in the following Dove Press journal:

Journal of Experimental Pharmacology

3 November 2009

Number of times this article has been viewed

\author{
Juan Arredondo' \\ Denys Omelchenko² \\ Alexander I Chernyavsky' \\ Jing Qian' \\ Maryna Skok ${ }^{2}$ \\ Sergei A Grando' \\ 'Institute for Immunology \\ and Departments of Dermatology \\ and Biological Chemistry, University \\ of California, Irvine, CA, USA; \\ ${ }^{2}$ Palladin Institute of Biochemistry, \\ Kiev, Ukraine
}

\begin{abstract}
We studied the involvement of nicotinic acetylcholine receptors (nAChRs) in the inflammation-related activity of human B-cell lines. Activation of nAChRs in Daudi cells with epibatidine abolished the pansorbin-dependent upregulation of the pro-inflammatory marker Cox-2 both at the mRNA and protein levels, indicating that the nicotinergic signaling suppresses B-cell activation. While the anti-inflammatory action on B-cells was mediated predominantly through $\alpha 7$ nAChR, as could be judged from abolishing epibatidine effects with methyllycaconitine, both $\alpha 7$ and non- $\alpha 7$ nAChRs, such as $\alpha 2$-containing receptors, were involved in regulation of B-cell apoptosis. The net effect was antiapoptotic. To determine the role of nAChRs in regulating B-cell activation/plasmacytic differentiation, we measured changes in the CD38, CD138 and Bcl-6 gene expression. Epibatidine significantly $(P<0.05)$ upregulated CD38 at the transcriptional level and CD138 and Bcl-6 - at the translational levels. AR-R17779 significantly $(P<0.05)$ increased the protein levels of CD38 and CD138. In both cases, the effect of epibatidine was abolished with Mec, and that of AR-R17779 - by MLA, demonstrating a functional role of $\mathrm{nAChRs}$ in regulating Daudi cell differentiation. The obtained results revealed distinct contributions of $\alpha 7$ and non- $\alpha 7$ nAChRs to regulation of B-cell activation/differentiation, and suggested that signaling through the nicotinic arm of acetylcholine regulatory axis is important for B-cell involvement in inflammation.
\end{abstract}

Keywords: Daudi B cell line, nicotinic acetylcholine receptor, inflammation, apoptosis, pansorbin

\section{Introduction}

Nicotinic acetylcholine receptors (nAChRs) are ligand-gated cation channels initially discovered in nerve and muscle cells and now being intensively studied in non-excitable cells of various origin. ${ }^{1}$ Structurally, nAChRs expressed in neuronal and non-neuronal cells are pentamers composed of different combinations of nine alpha $(\alpha 2-\alpha 10)$ and three beta $(\beta 2-\beta 4)$ subunits, with each subunit combination possessing certain pharmacologic sensitivity and regulating specific cell functions. ${ }^{2}$ The nAChRs expressed by immune cells can be activated by acetylcholine either released from innervating nerve terminals or endogenously produced and secreted by nonneuronal cells. ${ }^{3}$ Acetylcholine was reported to be proinflammatory for lymphocytes and epithelial cells, anti-inflammatory for mast cells and macrophages, both pro- and anti-inflammatory for monocytes, and variable for neutrophils and eosinophils. ${ }^{4}$ Stimulation of $\alpha 7 \mathrm{nAChR}$ with vagus-produced acetylcholine can attenuate the proinflammatory cytokine production. ${ }^{5,6}$ This receptor may mediate the anti-inflammatory effects of nicotine and other nAChR agonists. ${ }^{7}$ However, the
Correspondence: Sergei A Grando Institute for Immunology, University of California Irvine, I34 Sprague Hall, Irvine, CA 92697, USA

Tel + I 9498242713

Fax + I 9498242993

Email sgrando@uci.edu 
mechanisms by which nAChRs control inflammation and the cells involved in this process remain to be elucidated.

B lymphocytes are unique cells producing antibodies in response to antigenic stimuli. In addition, B-cells are considered the important players in regulating inflammation, because they can produce both proinflammatory (interleukin-6 [IL-6], tumor necrosis factor alpha [TNF $\alpha$ ], IL-12) and anti-inflammatory (IL-10) cytokines. ${ }^{8}$ Both normal B lymphocytes and some B lymphocyte-derived cell lines express various types of acetylcholine receptors. ${ }^{3}$ The nAChRs have been shown to regulate the development and survival of B lymphocytes. ${ }^{9,10}$ Nicotine stimulated proliferation of B lymphocyte-derived cell lines and normal plasma cells and decreased antibody production. ${ }^{11,12}$ Recent findings suggested an important role for $\alpha 7 \mathrm{nAChR}$ in the regulation of B-cell functions. Skok and colleagues ${ }^{13}$ found reduced preimmune level of serum immunoglobulin $G$ (IgG) in $\alpha 7$ knockout mice, whereas Fujii and colleagues ${ }^{14}$ demonstrated that the $\alpha 7 \mathrm{nAChR}$ expressed in murine immune cells exhibits negative regulation of antibody synthesis and controls the regulatory cytokine production. Stimulation of human leukemic B-cell lines expressing the $\alpha 2, \alpha 5, \alpha 6, \alpha 7, \alpha 9, \alpha 10$, and $\beta 2$ and $\beta 4 \mathrm{nAChR}$ subunits with nicotine or epibatidine elicited a transient $\mathrm{Ca}^{2+}$ signaling that was antagonized effectively by $\alpha$-bungarotoxin, suggesting that the $\alpha 7 \mathrm{nAChR}$ is, at least partly, responsible for nicotine-induced $\mathrm{Ca}^{2+}$ signaling in lymphocytes. ${ }^{3,15}$

The purpose of this study was to determine the involvement of $\alpha 7 v s$ non- $\alpha 7 \mathrm{nAChRs}$ in regulation of the inflammationand apoptosis-related events in human B cells.

\section{Materials and methods Cells and reagents}

The Daudi cells - a human Burkitt's lymphoma cell line - was purchased from ATCC (Catalog \# CCL-213) and grown in the ATCC complete growth medium (Catalog \#30-2001) at $37^{\circ} \mathrm{C}$ in a humid, $5 \% \mathrm{CO}_{2}$ incubator. Another Burkitt's lymphoma cell line, Ramos, as well as the line, $\mathrm{REH}$, corresponding to human pre-B lymphocytes were a kind gift of Dr Sidorenko (Kavetzky Institute of Experimental Oncology, Kiev, Ukraine). The cells were grown in RPMI-1640 medium supplemented with $20 \mathrm{mM}$ HEPES, $40 \mu \mathrm{g} / \mathrm{ml}$ gentamicin, and 10\% fetal bovine serum (SigmaAldrich, St. Louis, MO). The nicotinic ligands epibatidine, mecamylamine (Mec), methyllycaconitine (MLA) as well as 3-(4,5-dimethylthriasol-2-yl)-2,5-diphenyl tetrazolium bromide (MTT) were also from Sigma-Aldrich.
AR-R17779 was a gift from AstraZeneca Pharmaceuticals (Wilmington, DE). Pansorbin was purchased from EMD Chemicals, Inc. (Cat. No. 507862; Gibbstown, NJ). Fluorescein isothiocyanate (FITC)-labeled anti-CD19 (Cat. No. IM 1284) and phycoerythrin (PE)-labeled anti-CD22 antibodies (Cat. No. IM1835) were from Immunotech (Marseille, France). PE-labeled Streptavidin (Cat. No 554061) was from BD Biosciences (Bioline LCC, Kiev, Ukraine).

\section{Flow cytometry experiments}

The Daudi, Ramos, and REH cells were suspended to a concentration of $1 \times 10^{6}$ cells per tube in $50 \mu \mathrm{l}$ of $1 \%$ bovine serum albumin (BSA)-containing phosphate-buffered saline (PBS), and treated with biotinylated rabbit $\mathrm{nAChR}$ antibodies for $15 \mathrm{~min}$ at room temperature. The antibodies used in this study were raised against short synthetic fragments of the rat $\mathrm{nAChR}$ subunits $\alpha 4, \alpha 7, \beta 2$, or $\beta 4$ and shown to bind corresponding $\mathrm{nAChR}$ subunits in rat and guinea-pig autonomic ganglia ${ }^{16,17}$ and mouse B lymphocytes. ${ }^{10,11}$ The sequences used to generate the $\alpha 4, \beta 2$, and $\beta 4$ antibodies are identical between human and rat. Since the peptide used to raise anti- $\alpha 4$ antibody, AVGTYNTRKYEC, is highly homologous to the corresponding fragment of rat and human $\alpha 2$ subunit (ATGTYNSKKYDC; www.uniprot.org), this antibody also reacts with $\alpha 2 \mathrm{nAChR}$. The $\alpha 7$ antibody also recognizes human receptor because the peptide used for immunization contained only two conservative replacements: Lys for Arg and Ser for Asn. The specificity of $\alpha 7$ antibody was confirmed in experiments with $\alpha 7$ knockout mice (data not shown). The antibody concentration was selected according to ELISA with corresponding antigenic peptides, as detailed elsewhere. ${ }^{16,17}$ After a thorough wash with PBS, the cells were stained with PE-labeled streptavidin for $15 \mathrm{~min}$, washed by centrifugation and analyzed on the EPICS-XL flow cytometer (Coulter-Beckman, Fullerton, CA) using appropriate software. The cell aliquots stained with FITC-labeled anti-CD19 and PE-labeled anti-CD22 were analyzed similarly in a flow cytometer.

\section{Proliferation studies}

The Daudi, Ramos, and REH cells seeded in 96-well plates, $1.5 \times 10^{4}$ cells per well in $100 \mu \mathrm{l}$ of culture medium, were incubated with epibatidine at $37^{\circ} \mathrm{C}$ and $5 \% \mathrm{CO}_{2}$ for 48 hours. The number of live cells was measured by MTT inclusion according to Carmichael and colleagues. ${ }^{18}$ Briefly, MTT was added to the culture medium to the final concentration of $0.4 \mathrm{mg} / \mathrm{ml}$. After $4 \mathrm{hrs}$ of incubation at $37^{\circ} \mathrm{C}$, the medium was removed, and the formazan crystals formed were diluted in 
$100 \mu \mathrm{l}$ of dimethyl sulfoxide. After that, $25 \mu \mathrm{l}$ of $0.1 \mathrm{M}$ glycine, $0.1 \mathrm{M} \mathrm{NaCl}, \mathrm{pH}$ 10,5 were added to each well, and the absorbance at $545 \mathrm{~nm}$ was measured using StatFax 2100 microplate reader (Awareness Technology, Los Angeles, CA).

\section{Real-time quantitative polymerase chain reaction ( $q P C R$ ) experiments}

Total RNA was extracted from Daudi cells at the end of exposure experiments with the RNeasy Mini Kit (Qiagen, Valencia, CA) and used in the qPCR assay detailed elsewhere. ${ }^{19}$ All qPCR primers were designed with assistance from Primer Express software (version 2.0; Applied Biosystems, Foster City, CA) and the Assays-on-Demand service provided by Applied Biosystems. The qPCR reactions were performed using an ABI Prism 7500 Sequence Detection System (Applied Biosystems) and the TaqMan Universal Master Mix reagent (Applied Biosystems) in accordance to the manufacturer's protocol, as described by us in detail elsewhere. ${ }^{20}$ To correct for minor variations in mRNA extraction and reverse transcription, the gene expression values were normalized using the housekeeping gene glyceraldehyde-3-phosphate dehydrogenase. The data from triplicate samples were analyzed with a sequence detector software (Applied Biosystems), and results expressed as fold of control determined in intact (control) cells, and taken as 1 .

\section{In-cell Western (ICW) assay}

The ICW assay was performed as described by us in detail elsewhere $^{20}$ using reagents and equipment from LI-COR Biotechnology (Lincoln, NE). After incubation of $3 \times 10^{6}$ Daudi cell/well in a 96-well plate in culture media with or without test agents for 16 hours, the experimental and control Daudi cells were fixed in situ, washed, permeabilized with Triton solution, incubated with the LI-COR Odyssey Blocking Buffer for 1.5 hours and then treated overnight at $4{ }^{\circ} \mathrm{C}$ with a primary rabbit antibody to human cyclooxygenase-2 (Cox-2), Bcl-2, Bcl-6, Bax, CD38 or CD138 (all from Santa Cruz Biotechnology, Inc. Santa Cruz, CA). After that, the cells were washed and stained for one hour at room temperature with a secondary LI-COR IRDye $800 \mathrm{CW}$ goat antirabbit or IRDye $800 \mathrm{CW}$ anti-mouse antibodies, each diluted 1:800. Sapphire 700 (1:1000) was used to normalize for cell number/well. The protein expression was then quantitated using the LI-COR Odyssey Imaging System.

\section{Statistical analysis}

All experiments were performed in duplicates or triplicates, and the results were expressed as mean \pm standard deviation.
Statistical significance was determined using Student's $t$-test. Differences were deemed significant if the calculated $P$ value was $<0.05$.

\section{Results}

\section{The level of expression of nAChRs depends on B lymphocyte differentiation}

To select the most appropriate model for functional studies of $B$ cell nAChRs, we evaluated $n A C h R$ expression in three human $B$ cell lines representing different stages of B lymphocyte differentiation, ie, pre-B (REH), and mature B lymphocytes (Ramos) and B lymphoblasts (Daudi), using previously characterized rabbit affinity purified antibodies against the $\alpha 2 / \alpha 4, \alpha 7, \beta 2$, and $\beta 4$ subunits. ${ }^{16,17}$ The highest amount of antibody binding was observed in Daudi cells and the lowest in REH cells (Figure 1A). Because Daudi cells express $\alpha 2$, but not $\alpha 4$, subunit, ${ }^{3}$ the reactivity of $\alpha 2 / \alpha 4$ antibody was attributed to $\alpha 2 \mathrm{nAChR}$. Since Daudi cells are much larger than REH cells, and since each assay used the same number of cells, the higher fluorescence values could be due to a larger cell size. However, Ramos cells, which are similar in size to REH cells, also expressed significantly more $n A C h R s$ than REH cells both at the protein (Figure 1A) and mRNA (Figure 1B) levels, suggesting that an increased $\mathrm{nAChR}$ expression was associated with B-cell maturation rather than differences in cell size. Indeed, we found that Ramos cells expressed significantly more B lymphocyte differentiation markers CD19 and CD22 $2^{21}$ than REH cells (Figure 1C). Therefore, the B cell lines corresponding to mature human B lymphocytes expressed more nAChRs on their plasma membrane than pre-B-cells. However, the two cell lines, Daudi and Ramos, both related to a similar type of malignancy (Burkitt's lymphoma) expressed different relative amounts of distinct nAChR subtypes. While in Daudi cells the ratio of the signals produced by $\alpha 2 / \alpha 4$ vs $\alpha 7$ antibodies was 2.8, that in Ramos cells was 9.4, indicating that the former cell type possesses a relatively higher level of $\alpha 7 \mathrm{nAChRs}$. Since $\alpha 7$ had been reported to play a major role in mediating acetylcholine effects on inflammation, ${ }^{6,14,22,23}$ we selected Daudi cells for the functional studies described below.

\section{Nicotinic signaling modulates the effects of pansorbin on the inflammatory and apoptosis markers in Daudi cells}

To elucidate the role of $\mathrm{nAChRs}$ in the inflammation-related function of B lymphocytes, we studied the nicotinergic effects on the expression of inflammatory and apoptotic 

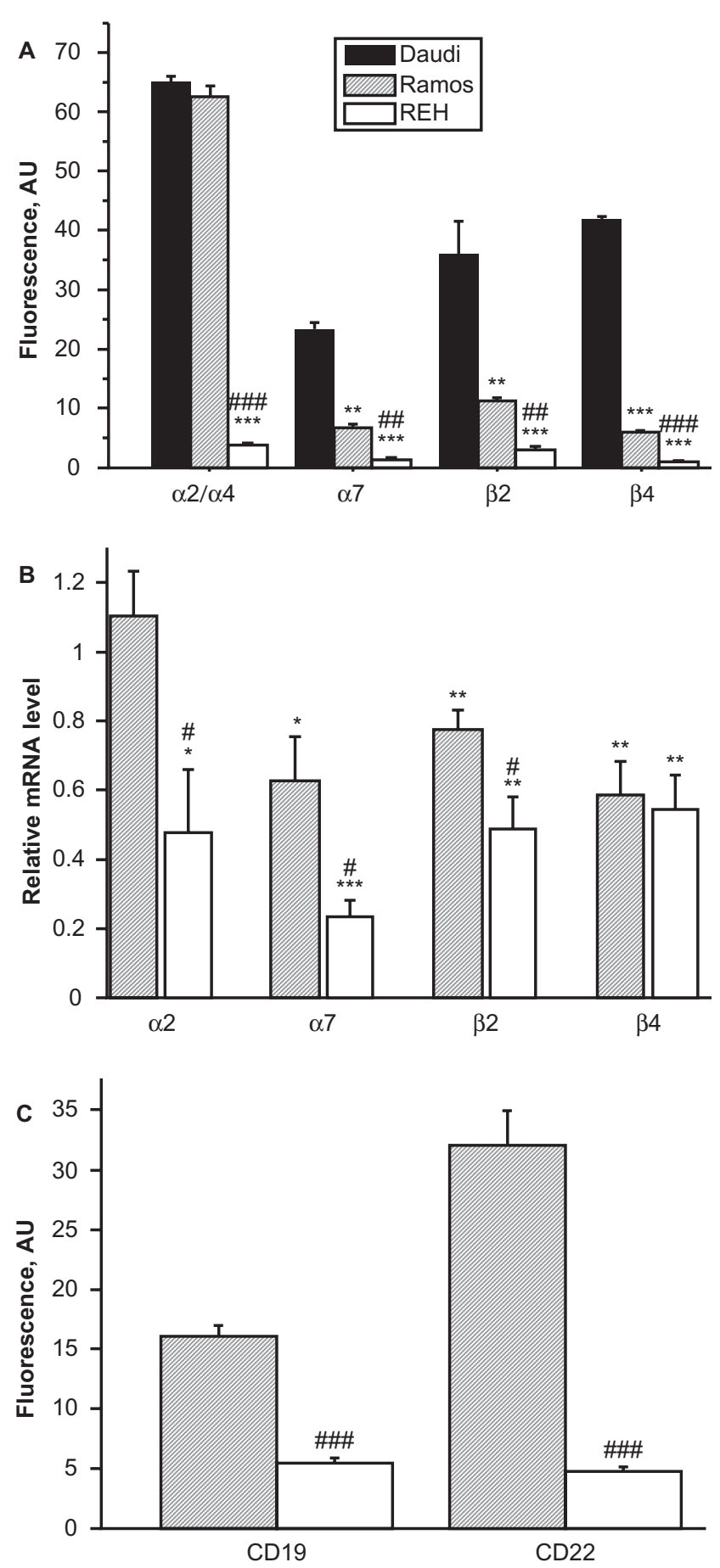

Figure I Expression of $n A C h R$ subunits and $B$ cell differentiation markers in Daudi, Ramos, and REH cells. A) The Daudi, Ramos, and REH cells, $I \times 10^{6}$ cells per tube in $50 \mu \mathrm{l}$ of $1 \%$ BSA-containing PBS, were treated with rabbit $\alpha 2 / \alpha 4-, \alpha 7-, \beta 2-$, or $\beta 4$-specific antibodies and the antibody binding was analyzed by flow cytometry as detailed in the Materials and methods section. B) qPCR analysis of equal amounts of cDNA from Daudi, Ramos and REH cells using the procedure described in Materials and methods. Results are fold of control values determined in Daudi cells, and taken as I.C) Ramos and REH cells were stained with FITC-labeled anti-CD I 9 or PE-labeled anti-CD22 and analyzed by flow cytometry.

Notes: $* P<0.005$, $* * P<0.005$, and $* * * P<0.0005$ compared to Daudi cells; ${ }^{\# P}<0.005,{ }^{\# P}<0.005$, and ${ }^{\# \# P}<0.0005$ compared to Ramos cells.

Abbreviations: $A U$, arbitrary units; BSA, bovine serum albumin; FITC, fluorescein isothiocyanate; $n A C h R s$, nicotinic acetylcholine receptors; PBS, phosphate-buffered saline; $\mathrm{PE}$, phycoerythrin; $\mathrm{QPCR}$, quantitive polymerase chain reaction. markers in Daudi cells activated with pansorbin. It is well established that activation of human B cells upregulates the inducible Cox-2 isoenzyme, ${ }^{24}$ a marker of inflammation, and alters the expression/distribution of the apoptosis related proteins of the Bcl-2 family. ${ }^{25,26}$ To activate B cells, we employed pansorbin, ie, heat-killed and fixed Staphylococcus aureus cells stimulating the mitogenic response of B lymphocytes..$^{27}$ First, we sought to confirm that pansorbin stimulation alters expression of the genes encoding the proinflammatory marker Cox-2, and the anti- and proapoptotic markers Bcl-2 and Bax, respectively, at the RNA and protein levels. As expected, stimulation of Daudi cells with $0.05 \%$ pansorbin caused a several fold increase of the Cox-2 mRNA and protein contents (Figure 2), indicating that pansorbin exhibits a pro-inflammatory action. The apoptosis-related effect of pansorbin was complex: it significantly $(P<0.05)$ upregulated the Bcl-2 mRNA and protein levels but also increased by almost fivefold the level of Bax protein, whereas the Bax mRNA level did not change compared to control $(P>0.05)$. These results indicated that the amounts of $\mathrm{Bax}$ and $\mathrm{Bcl}-2$ proteins were regulated by pansorbin in different ways. An increase of Bcl-2 was caused by upregulated transcription, whereas that of Bax was induced at the post-transcriptional level. Since the increase of Bax protein was evidently stronger than that of Bcl-2, the net effect of pansorbin was proapoptotic.

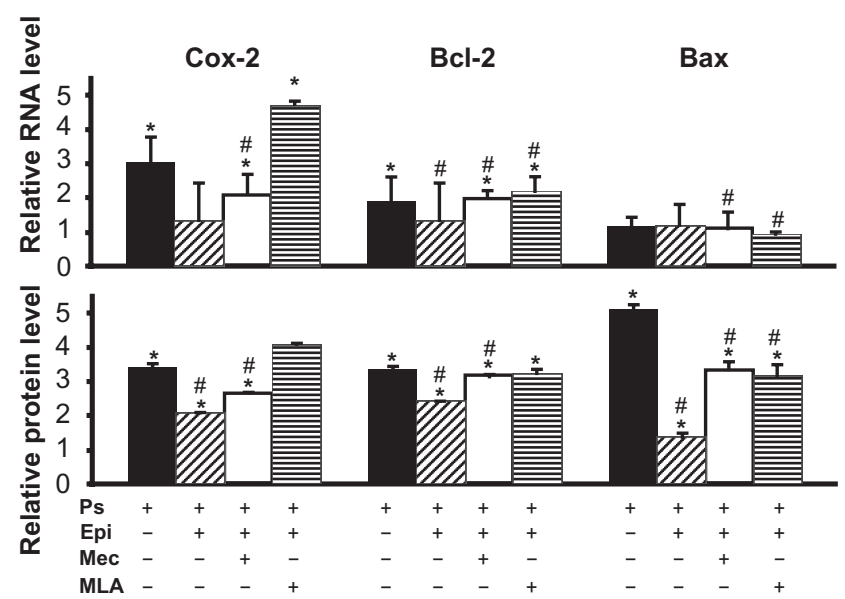

Figure 2 Nicotinic effects on pansorbin-induced activation of Daudi cells. Daudi cells, $3 \times 10^{6}$ cells/well, were incubated for 16 hours in a humid, $5 \% \mathrm{CO}_{2}$ incubator in the culture medium containing $0.05 \%$ pansorbin (Ps) in the absence or presence of I $\mu \mathrm{M}$ epibatidine (Epi) $\pm 50 \mu \mathrm{M}$ Mec or $100 \mathrm{nM}$ MLA, after which the expression of the genes encoding Cox-2, Bcl-2, and Bax at the mRNA and protein levels was measured by qPCR and ICW, respectively, as detailed in Materials and methods. The results are expressed as fold of control values determined in intact Daudi cells taken as I.

Notes: $* P<0.05$ compared to control values; $E P<0.05$ compared to pansorbin given alone.

Abbreviations: ICW, in-cell Western blotting; Mec, mecamylamine; MLA, methyllycaconitine; qPCR, quantitive polymerase chain reaction. 
To elucidate the role of nAChRs in the proinflammatory and proapoptotic responses of Daudi cells, we exposed the cells to pansorbin in the presence of $1 \mu \mathrm{M}$ epibatidine, a nonselective nicotinic agonist. ${ }^{28,29}$ We used the $1 \mu \mathrm{M}$ concentration of epibatidine to activate all nAChR subtypes expressed in Daudi cells. Epibatidine abolished the pansorbin-induced upregulation of all studied biomarkers (Figure 2). While Cox-2 expression was suppressed at both mRNA and protein levels, a significant $(P<0.05)$ inhibition of pansorbin-induced Bcl-2 and Bax upregulation was observed only at the protein level. Since the decrease of Bax protein was much stronger than that of Bcl-2, the net effects of epibatidine was antiapoptotic. These findings indicated that nicotinergic signaling in B lymphocytes is both antiinflammatory and antiapoptotic.

To estimate the relative contribution of $\alpha 7 \mathrm{nAChRs}$ to the observed effects of epibatidine, we applied MLA, a competitive inhibitor of $\alpha 7$-containing nAChRs. ${ }^{30} \mathrm{We}$ also used Mec, a channel blocker that can inhibit $\alpha 7$ as well as non- $\alpha 7 \mathrm{nAChR}$ subtypes such as $\alpha 2 .^{28}$ The effect of epibatidine on the pansorbin-induced elevation of Cox-2 was abolished completely by MLA and, to a lesser extent, by Mec (Figure 2). In contrast, both antagonists were equally efficient in reducing the inhibitory effect of epibatidine on the pansorbin-dependent increase of Bcl-2 and Bax in Daudi cells (Figure 2).

These findings suggested that the anti-inflammatory nicotinergic signaling in B lymphocyte-derived cells is mediated predominantly through the $\alpha 7$-containing nAChRs, whereas apoptotic events are regulated through both $\alpha 7$ and non- $\alpha 7$ nAChRs.

\section{The nicotinergic effects on the Daudi cell plasmacytic differentiation}

To determine the role of the nicotinergic stimulation on regulation of B lymphocyte proliferation, we exposed Daudi cells to 1 or $10 \mu \mathrm{M}$ epibatidine and measured the MTT inclusion after 48 hours of incubation. No statistically significant changes could be detected (data not shown).

Next, we measured changes in the relative amounts of mRNAs and proteins of markers of activation/plasmacytic differentiation in the Daudi cells exposed to epibatidine $\pm \mathrm{Mec}$, to activate/inactivate all nAChRs, or AR-R17779 \pm MLA, to selectively activate/inactivate $\alpha 7 \mathrm{nAChR}{ }^{31,32}$ The exposed cells where used in the qPCR and ICW assays of CD38, CD138 and Bcl- 6 that had been previously identified as sensitive biomarkers of the Daudi cell state. ${ }^{33}$ In most cases, both epibatidine and AR-R17779 produced only moderate changes on the gene expression (Figure 3). However, epibatidine significantly $(P<0.05)$ upregulated CD38 at the transcriptional level and CD138 and Bcl-6 at the translational levels. AR-R17779 significantly $(P<0.05)$ increased the protein levels of CD38 and CD138 (Figure 3). In both cases, the effect of epibatidine was abolished with Mec, and that of ARR17779 by MLA. These results demonstrated a functional role of nAChRs in regulating Daudi cells differentiation and revealed distinct contribution of $\alpha 7$ and non- $\alpha 7 \mathrm{nAChRs}$ in this process.

\section{Discussion}

In this study, we used human B lymphocyte-derived cell lines to investigate the role of $\alpha 7$ and non- $\alpha 7 \mathrm{nAChRs}$ in regulation of B-cell involvement in inflammation. Among the three B-cell lines tested, we selected Daudi cells because they appeared to express on their cell surfaces the highest amounts of nAChRs with a relatively high ratio of $\alpha 7$ to non- $\alpha 7$ nAChRs. Comparison of Ramos and REH cells demonstrated that the nAChR expression increases with B-cell maturation. These findings suggested that signaling through the nicotinic arm of acetylcholine regulatory axis is critical for maturation of B lymphocytes. This is in accord with our previously published data on mouse B lymphocytes. ${ }^{9}$

We showed for the first time that the nAChRs expressed by Daudi cells are coupled to an anti-inflammatory function, as nAChR activation with epibatidine inhibited pansorbin-induced upregulated Cox-2 expression.

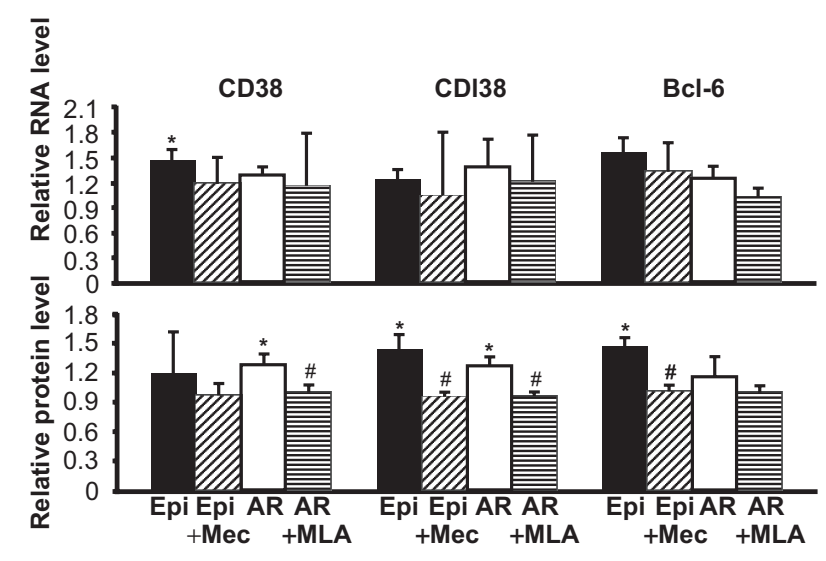

Figure 3 Nicotinic effects on the expression of differentiation markers in Daudi cells. Daudi cells, $3 \times 10^{6}$ cells/well, were incubated with I $\mu \mathrm{M}$ epibatidine (Epi) $\pm 50 \mu \mathrm{M} \mathrm{Mec}$ or $100 \mu$ MAR-R 17779 (AR) $\pm 100 \mathrm{nM} \mathrm{MLA}$, after which the expression of the genes encoding CD38, CDI38, and $\mathrm{Bcl}-6$ at the mRNA and protein levels was measured by qPCR and ICW, respectively, as detailed in the Materials and methods. The results are expressed as fold of control values determined in intact Daudi cells.

Notes: $* P<0.05$ compared to control values; $€ P<0.05$ compared to a relevant agonist given alone.

Abbreviations: ICW, in-cell Western blotting; Mec, mecamylamine; MLA, methyllycaconitine; qPCR, quantitive polymerase chain reaction. 
In keeping with the notion that $\alpha 7$ is coupled to suppression of inflammation, ${ }^{6,14,22,23}$ the $\alpha 7$ selective antagonist MLA was more efficient than the nonselective antagonist $\mathrm{Mec}$ in abolishing the suppressive action of epibatidine on Cox-2 upregulation. We cannot ignore a possibility that some of the MLA effects were mediated by $\alpha 9$-made $\mathrm{nAChR}$, but its expression in Daudi cells remains to be elucidated. Our data seem to disagree with the reports that nAChR signaling is pro-inflammatory in lymphocytes. ${ }^{34,35}$ However, in contrast to the anti-inflammatory action of $\alpha 7 \mathrm{nAChR}$, the proinflammatory cascade is believed to be activated through the pathways coupled by other nAChRs. ${ }^{36}$

The nicotinergic stimulation also considerably altered the pansorbin-induced expression of the genes regulating apoptosis of Daudi cells. as could be expected due to the fact that cellular processes mediating lymphocyte activation, inflammation, and cell death are interrelated. Since epibatidine abolished the elevation of Bax over Bcl-2 by pansorbin, and since both Mec and MLA antagonized epibatidine effects equally efficiently, we believe that the net nicotinergic effect was antiapoptotic in Daudi cells, and that both $\alpha 7$ and non- $\alpha 7$ nAChRs were involved. This is in keeping with a previous report that the $\beta 2$ - and $\alpha 7-$ knockout mice show increased numbers of apoptotic B cells within the bone marrow. ${ }^{9}$ The nAChR activation, however, did not affect Daudi cell proliferation, which may be explained by a strong proliferation potential of malignant cells.

The expression of cell activation/differentiation markers, such as CD38, CD138, and Bcl-6, was also sensitive to regulation through $n A C h R s$. Stimulation of Daudi cells with the $\alpha 7$ agonist AR-R17779 upregulated expression of CD38 and CD138, but had no effect of Bcl-6 that, however, could be elevated by epibatidine. These observations highlighted a differential role of $\alpha 7$ and non- $\alpha 7$ nAChRs in cholinergic control of activation/plasmacytic differentiation of Daudi cells. CD38 is an ADP-ribosyl cyclase which also functions in cell adhesion, signal transduction and calcium signaling. It is considered as a marker of chronic B lymphocyte leukaemia and its receptor functions are connected to increased proliferative potential of B lymphocytes. ${ }^{37} \mathrm{CD} 138$ is a transmembrane (type I) heparan sulfate proteoglycan which is considered a marker of B lymphocyte plasmacytic differentiation. ${ }^{38}$ BCL6 acts as a sequence-specific repressor of transcription, and its concomitant downregulation is required for completion of the plasma cell differentiation program. ${ }^{39}$

In conclusion, results of the present study emphasized an important role of the acetylcholine regulatory axis in B cell development and function. The obtained data demonstrated that nAChR signaling interferes with intracellular pathways triggered by $B$ cell activation. Both $\alpha 7$ and non- $\alpha 7$ nAChRs are involved but fulfill different functions. Activation of distinct $\mathrm{nAChR}$ subtypes during B-cell development may exhibit differential effects on B-cell survival/activity. The unique coupling to regulation of a distinct cell function of nAChR subtypes expressed at different stages of B-cell development can diversify the immunoregulatory effects of the auto/paracrine cytotransmitter acetylcholine allowing it to coordinate the immune response to a specific environmental stimulus. Further elucidation of the acetylcholine receptor subtypes coupled to regulation of specific B cell functions will help in developing novel immunomodulatory therapies with nicotinic drugs allowing selective immunocorrection.

\section{Acknowledgments}

This work was supported by the NIH grants ES014384 and DE14173, and research grants from the Institute for Science and Health (to SAG), and Philip Morris USA Inc. and Philip Morris International (to MVS and DMO). The authors report no conflicts of interest in this work.

\section{References}

1. Wessler I, Kirkpatrick CJ. Acetylcholine beyond neurons: the non-neuronal cholinergic system in humans. Br J Pharmacol. 2008;154:1558-1571.

2. Paterson D, Nordberg A. Neuronal nicotinic receptors in the human brain. Prog Neurobiol. 2000;61:75-111.

3. Kawashima K, Fujii T. Expression of non-neuronal acetylcholine in lymphocytes and its contribution to the regulation of immune function. Front Biosci. 2004;9:2063-2085.

4. Gwilt CR, Donnelly LE, Rogers DF. The non-neuronal cholinergic system in the airways: an unappreciated regulatory role in pulmonary inflammation? Pharmacol Ther. 2007;115:208-222.

5. Borovikova LV, Ivanova S, Zhang M, et al. Vagus nerve stimulation attenuates the systemic inflammatory response to endotoxin. Nature. 2000;405:458-462.

6. Wang H, Yu M, Ochani M, et al. Nicotinic acetylcholine receptor alpha7 subunit is an essential regulator of inflammation. Nature. 2003;421:384-388.

7. de Jonge WJ, Ulloa L. The alpha7 nicotinic acetylcholine receptor as a pharmacological target for inflammation. Br J Pharmacol. 2007;151:915-929.

8. Hasan M, Lopez-Herrera G, Blomberg KE, et al. Defective Toll-like receptor 9-mediated cytokine production in B cells from Bruton's tyrosine kinase-deficient mice. Immunology. 2008;123:239-249.

9. Skok M, Grailhe R, Agenes F, Changeux JP. The role of nicotinic acetylcholine receptors in lymphocyte development. J Neuroimmunol. 2006;171:86-98.

10. Skok MV, Grailhe R, Agenes F, Changeux JP. The role of nicotinic receptors in B-lymphocyte development and activation. Life Sci. 2007;80:2334-2336.

11. Skok MV, Kalashnik EN, Koval LN, et al. Functional nicotinic acetylcholine receptors are expressed in B lymphocyte-derived cell lines. Mol Pharmacol. 2003;64:885-889.

12. Koval LM, Romaniuk SI, Kolybo DV, Skok MV, Komisarenko SV. The role of nicotine in regulation of lymphocyte proliferation. Ukr Biokhim Zh. 2005;77:105-111. 
13. Skok M, Grailhe R, Changeux JP. Nicotinic receptors regulate B lymphocyte activation and immune response. Eur J Pharmacol. 2005;517:246-251.

14. Fujii YX, Fujigaya H, Moriwaki Y, et al. Enhanced serum antigenspecific IgG1 and proinflammatory cytokine production in nicotinic acetylcholine receptor alpha 7 subunit gene knockout mice. JNeuroimmunol. 2007;189:69-74.

15. Kimura R, Ushiyama N, Fujii T, Kawashima K. Nicotine-induced $\mathrm{Ca}^{2+}$ signaling and down-regulation of nicotinic acetylcholine receptor subunit expression in the CEM human leukemic T-cell line. Life Sci. 2003;72:2155-2158

16. Skok MV, Voitenko LP, Voitenko SV, et al. Alpha subunit composition of nicotinic acetylcholine receptors in the rat autonomic ganglia neurons as determined with subunit-specific anti-alpha(181-192) peptide antibodies. Neuroscience. 1999;93:1427-1436.

17. Koval OM, Voitenko LP, Skok MV, et al. The $\beta$-subunit composition of nicotinic acetylcholine receptors in the neurons of the guinea pig inferior mesenteric ganglion. Neurosci Lett. 2004;365:143-146.

18. Carmichael J, DeGraff WG, Gazdar AF, Minna JD, Mitchell JB. Evaluation of a tetrazolium-based semiautomated colorimetric assay: assessment of radiosensitivity. Cancer Res. 1987;47:943-946.

19. Arredondo J, Chernyavsky AI, Marubio LM, et al. Receptor-mediated tobacco toxicity: Regulation of gene expression through $\alpha 3 \beta 2$ nicotinic receptor in oral epithelial cells. Am J Pathol. 2005;166:597-613.

20. Arredondo J, Chernyavsky AI, Jolkovsky DL, Webber RJ, Grando SA SLURP-2: A novel cholinergic signaling peptide in human mucocutaneous epithelium. J Cell Physiol. 2006;208:238-245.

21. Lucio P, Parreira A, van den Beemd MW, et al. Flow cytometric analysis of normal B cell differentiation: a frame of reference for the detection of minimal residual disease in precursor-B-ALL. Leukemia. 1999;13:419-427.

22. Yoshikawa H, Kurokawa M, Ozaki N, et al. Nicotine inhibits the production of proinflammatory mediators in human monocytes by suppression of I-kappaB phosphorylation and nuclear factor-kappaB transcriptional activity through nicotinic acetylcholine receptor alpha7. Clin Exp Immunol. 2006;146:116-123.

23. Hamano R, Takahashi HK, Iwagaki H, Yoshino T, Nishibori M, Tanaka N. Stimulation of alpha7 nicotinic acetylcholine receptor inhibits CD14 and the toll-like receptor 4 expression in human monocytes. Shock. 2006;26:358-364.

24. Ryan EP, Pollock SJ, Murant TI, Bernstein SH, Felgar RE, Phipps RP. Activated human B lymphocytes express cyclooxygenase-2 and cyclooxygenase inhibitors attenuate antibody production. J Immunol. 2005;174:2619-2626.

25. Grillot DA, Merino R, Pena JC, et al. bcl-x exhibits regulated expression during B cell development and activation and modulates lymphocyte survival in transgenic mice. J Exp Med. 1996;183:381-391.

26. Souvannavong V, Lemaire C, Chaby R. Lipopolysaccharide protects primary B lymphocytes from apoptosis by preventing mitochondrial dysfunction and bax translocation to mitochondria. Infect Immun. 2004;72:3260-3266.
27. Kierszenbaum F, Moretti E, Sztein MB. Trypanosoma cruzi induces suppression of DNA synthesis and inhibits expression of interleukin-2 receptors by stimulated human B lymphocytes. Immunology. 1991;74:317-322.

28. Stauderman KA, Mahaffy LS, Akong M, et al. Characterization of human recombinant neuronal nicotinic acetylcholine receptor subunit combinations alpha2beta4, alpha3beta4 and alpha4beta4 stably expressed in HEK293 cells. J Pharmacol Exp Ther. 1998;284:777-789.

29. Wang F, Gerzanich V, Wells GB, et al. Assembly of human neuronal nicotinic receptor $\alpha 5$ subunits with $\alpha 3, \beta 2$, and $\beta 4$ subunits. $J$ Biol Chem. 1996;271:17656-17665.

30. Alexander SP, Mathie A, Peters JA. Guide to receptors and channels, 1st edition (2005 revision). Br J Pharmacol. 2005;144(Suppl 1):S1-128.

31. Mullen G, Napier J, Balestra M, et al. (-)-Spiro[1-azabicyclo[2.2.2]octane3,5'-oxazolidin-2'-one], a conformationally restricted analogue of acetylcholine, is a highly selective full agonist at the alpha 7 nicotinic acetylcholine receptor. J Med Chem. 2000;43:4045-4050.

32. Macor JE, Mullen G, Verhoest P, Sampognaro A, Shepardson B, Mack RA. A chiral synthesis of (-)-spiro[1-azabicyclo[2.2.2] octane3,5'- oxazolidin-2'-one]: a conformationally restricted analogue of acetylcholine that is a potent and selective alpha7 nicotinic receptor agonist. J Org Chem. 2004;69:6493-6495.

33. Teng Y, Takahashi Y, Yamada M, et al. IRF4 negatively regulates proliferation of germinal center B cell-derived Burkitt's lymphoma cell lines and induces differentiation toward plasma cells. Eur J Cell Biol. 2007;86:581-589.

34. Aicher A, Heeschen C, Mohaupt M, Cooke JP, Zeiher AM, Dimmeler S. Nicotine strongly activates dendritic cell-mediated adaptive immunity: potential role for progression of atherosclerotic lesions. Circulation. 2003;107:604-611.

35. Hallquist N, Hakki A, Wecker L, Friedman H, Pross S. Differential effects of nicotine and aging on splenocyte proliferation and the production of Th1- versus Th2-type cytokines. Proc Soc Exp Biol Med. 2000;224:141-146.

36. Vincler M, Vetter DE, McIntosh M. $\alpha 9 \alpha 10$ and $\alpha 7$ nicotinic acetylcholine receptors have opposite roles in the immune response to peripheral nerve injury. Biochem Pharmacol. 2007;74:SMA49 (45.41).

37. Deaglio S, Capobianco A, Bergui L, et al. CD38 is a signaling molecule in B-cell chronic lymphocytic leukemia cells. Blood. 2003;102: 2146-2155.

38. Ozcan E, Garibyan L, Lee JJ, Bram RJ, Lam KP, Geha RS. Transmembrane activator, calcium modulator, and cyclophilin ligand interactor drives plasma cell differentiation in LPS-activated B cells. J Allergy Clin Immunol. 2009;123:1277-1286 e1275.

39. Diehl SA, Schmidlin H, Nagasawa M, et al. STAT3-mediated up-regulation of BLIMP1 Is coordinated with BCL6 down-regulation to control human plasma cell differentiation. J Immunol. 2008;180 4805-4815.
Journal of Experimental Pharmacology

\section{Publish your work in this journal}

The Journal of Experimental Pharmacology is an international, peerreviewed, open access journal publishing original research, reports, reviews and commentaries on all areas of laboratory and experimental pharmacology. The manuscript management system is completely online and includes a very quick and fair peer-review system.

\section{Dovepress}

Submit your manuscript here: http://www.dovepress.com/journal-of-experimental-pharmacology-journal 